\title{
Efficacy of different analgesic or sedative drug therapies in pediatric patients with congenital heart disease undergoing surgery: a network meta-analysis
}

\author{
Rui-Zhu Liu' ${ }^{1}$ Bing-Tong $\mathrm{Li}^{2}$ · Guo-Qing Zhao ${ }^{1}$ (I) \\ Received: 19 October 2018 / Accepted: 27 March 2019 / Published online: 23 April 2019 \\ ๑) Children's Hospital, Zhejiang University School of Medicine 2019
}

\begin{abstract}
Background Surgery is an effective therapy for congenital heart disease (CHD) and the management after surgery poses challenges for the clinical workers. We performed this network meta-analysis to enhance the corresponding evidence with respect to the relative efficacy of different drug treatments applied after the CHD surgery.

Methods Embase and PubMed were systematically retrieved to identify all published controlled trials investigating the effectiveness of drugs for patients up to 25 August, 2018. Mean differences (MD), odds ratios and their 95\% credible intervals (CrIs) were used to evaluate multi-aspect comparisons. Surface under cumulative ranking curve (SUCRA) was used to analyze the relative ranking of different treatments in each endpoint.

Results Compared to saline, all the drugs achieved better preference under the efficacy endpoints except fentanyl in JET. As for ventilator time, all drugs were more effective than saline while only the difference of dexmedetomidine was statistically obvious ( $\mathrm{MD}=6.92,95 \%$ CrIs 1.77-12.54). Under the endpoint of ICU time, dexmedetomidine was superior to saline as well ( $\mathrm{MD}=1.26,95 \%$ CrIs $0.11-2.45)$. When all the endpoints were taken into consideration and with the help of ranking probabilities and SUCRA values, fentanyl combined with dexmedetomidine was one of the recommended drugs due to its shorter time on ventilator and stay in hospital as well as lower mortality.

Conclusions Overall, based on the comprehensive consideration of all the endpoints, fentanyl combined with dexmedetomidine was considered to be the best-recommended clinical interventions among all the methods.
\end{abstract}

Keywords Congenital heart disease $\cdot$ Dexmedetomidine $\cdot$ Drug treatments $\cdot$ Fentanyl $\cdot$ Network meta-analysis

\section{Introduction}

Congenital heart diseases (CHD) arise from abnormal formation of the heart or major blood vessels [1]. There is $0.4-1$ infants born with congenital heart disease in per 100 US infants and approximately $1 / 4$ of these lesions will

Electronic supplementary material The online version of this article (https://doi.org/10.1007/s12519-019-00252-4) contains supplementary material, which is available to authorized users.

Guo-Qing Zhao

zhaogq65@126.com

1 Department of Anesthesiology, China-Japan Union Hospital of Jilin University, Changchun 130000, China

2 Department of Rheumatology and Immunology, China-Japan Union Hospital of Jilin University, Changchun 130000, China require intervention, such as surgery, during infancy [1,2]. Approximately 10,000 children require anesthesia every year for CHD surgery during their first year of life [3]. Pre- and postoperative treatment of children with CHD poses enormous challenges in the intensive care unit (ICU), especially during the postoperative phase $[4,5]$.

Intravenous benzodiazepines and opioids are the most commonly used drugs for sedation and analgesia in the pediatric ICU [6]. Fentanyl is the most commonly used opioid for procedural sedation because of its pharmacokinetic profile, analgesic potency, and low cost [7]. The combination of benzodiazepines and opioids often provide satisfactory sedation of children; however, they are associated with adverse reactions such as respiratory depression, prolonged mechanical ventilation, and prolonged ICU stay $[8,9]$. Dexmedetomidine (DEX) is a selective alpha-2-adrenergic receptor antagonist that possesses sedative, anxiolytic, and analgesic effects without causing significant respiratory depression 
$[10,11]$. Thus, a lot of previous studies have focused on the comparison of fentanyl and dexmedetomidine. In addition, few studies reported the efficacy comparison between dexmedetomidine and other kinds of standard analgesia and sedation medications such as drugs combination consisted of midazolam, propofol, buprenorphine and pentazocine [12].

Junctional ectopic tachycardia (JET) is one type of arrhythmia encountered postoperatively, and is observed almost exclusively after open heart surgery in children with CHD [13]. Patients who developed JET postoperatively tend to have prolonged intensive care unit (ICU) stay. Hemodynamic instability as a result of JET leads to increased mortality [14]. Recently, perioperative administration of dexmedetomidine had been studied as an alternative approach in the treatment of postoperative JET.

Although varieties of the studies focused on the pairwise comparison and improving the precision of estimates results, they cannot fully satisfy the comparison requirement. Besides, a majority of current systematic analyses were restricted to only one medication in terms of a very few endpoints [15-17]. In this study, we conducted a comprehensive search strategy aiming to include all the available drugs and data extracted from the controlled trails to compare and evaluate their relative efficacy at the same time. A total of seven kinds of different therapy methods (saline, dexmedetomidine alone, dexmedetomidine combined with fentanyl, fentanyl alone, analgesic and sedative, etomidate and sufentanil) have been involved in this NMA. Five outcomes have been considered: time on ventilator (VT), time in intensive care unit (ICU), length of stay (LOS), junctional ectopic tachycardia (JET) and mortality. The aim of this NMA is to expand the existing studies and with regard to the results which were obtained from this study, we tried to identify the optimal drugs for the postoperative patients with CHD.

\section{Methods}

\section{Study design and selection strategy}

The database Embase and PubMed were systematically retrieved to identify all published experimental studies, investigating the outcomes of different analgesic or sedative drug therapies in pediatric patients undergoing congenital heart disease surgery up to 25 August, 2018. The medical subject headings, "drug therapy", "control trail", "randomized control trial", "observational study" and their synonyms were combined as search field. The related bibliographies were also searched manually to identify studies that were missed in the electronic search. The search was limited to clinical trials and human studies, age restrictions and language were not implemented.

\section{Inclusion criteria}

A large scale of papers and studies selected for our network meta-analysis (NMA) were evaluated if they met the following standards: (1) patients enrolled in this study were infant or child with congenital heart disease and going through surgery; (2) study should at least include two of the eight treatments; (3) study should at least include one of the following outcomes, i.e., time on ventilator (VT), intensive care unit stay (ICU), length of stay in hospital (LOS), and junctional ectopic tachycardia (JET); and (4) original trials should comprise adequate data for NMA.

\section{Exclusion criteria}

Studies will be excluded if they satisfy any of the following standards: (1) studies have no primary endpoint; (2) studies focus on other relevant treatments; (3) replicated or incomplete data from the same cohort; (4) the publication type of study are meetings, case reports, and publication reviews.

\section{Outcome measures and data extraction}

All relevant data from enrolled studies including references of all retrieved publications were extracted independently by two reviewers using a standardized data collection form. Discrepancies between investigators were resolved by consensus, referring back to eligible study or discussed through a third independent investigator. In this NMA analysis, VT, ICU and LOS were considered as the primary outcomes, and JET and mortality were regarded as secondary outcomes. The following publication information was extracted from each study: (1) the first author's last name, year of publication and country; (2) study design, blind mode, the specific sort of CHD, anesthetic maintained drug and the therapeutic drugs and dosages after surgery; (3) participants number and several demographic characteristics; and (4) the outcomes contained in the study.

\section{Statistical analysis}

The statistical processing was carried out by $\mathrm{R}$ (Version 3.5.1) and STATA version 13.0 (Stata Corp, College Station, TX, USA). The first-hand correlation between two interventions, among VT, ICU, LOS, JET and mortality were displayed in the network mapping. The dot sizes illustrated the number of patients receiving corresponding therapy, and the width of lines indicated the number of each comparison. NMA was based on Bayesian framework using a random-effects model. In principle, through NMA, we could randomly allocate any two of the interventions under the specific endpoint. For continuous variables, such as VT, ICU time and LOS, the MD with its $95 \% \mathrm{CrI}$ was measured while 
Fig. 1 Flow diagram summarizing results of study identification and selection

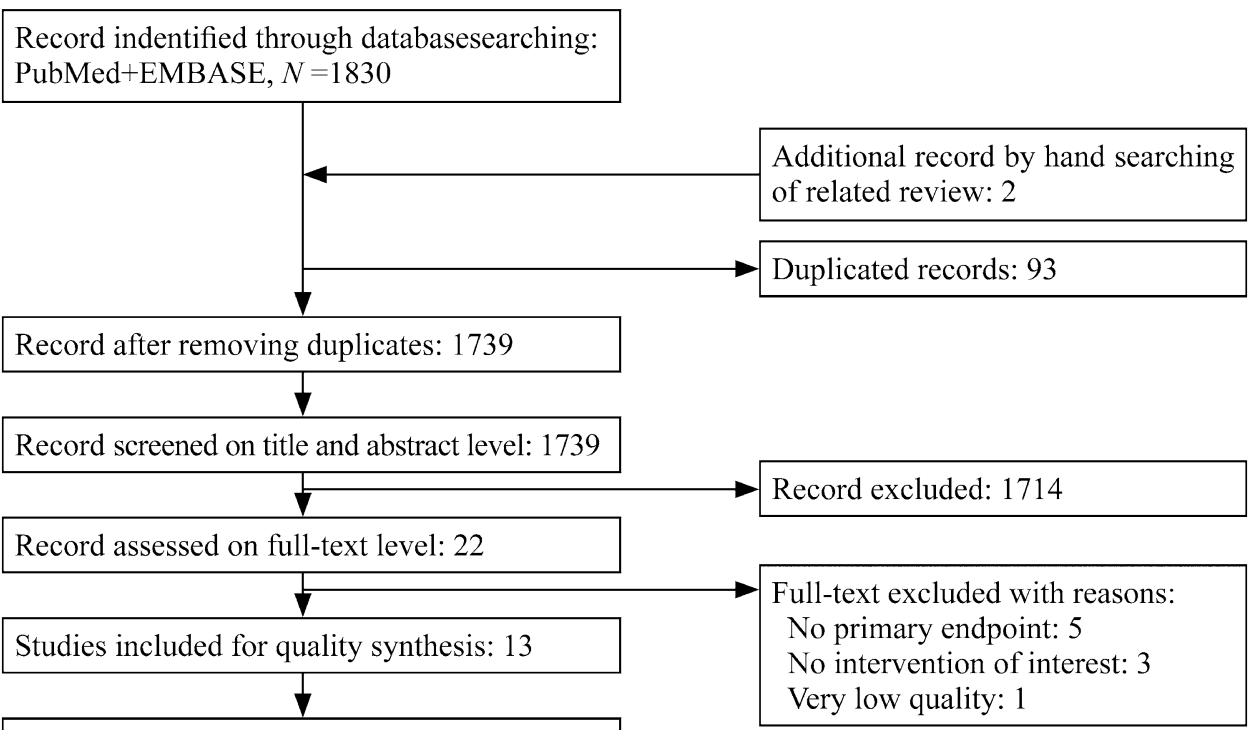

Studies included for quantitative synthesis: 13 the binary variables, such as morality, were represented as odds ratio (OR) with 95\% CrI. Furthermore, surface under cumulative ranking curve (SUCRA) was used to analyze the relative ranking of different treatments in each endpoint. The values of SUCRA are positively correlated with outcomes and the maximum ranking value is 1 . Additionally, net heat plots were conducted to exhibit the degree of inconsistency. Finally, comparison-adjusted funnel plots were used to evaluate publication bias. A symmetry plot indicated that there are no small sample sizes affecting the statistical results.

\section{Results}

\section{Study selection}

Figure 1 showed the process of searching and screening. First, a total of 1830 records were identified through searching methods mentioned early. Another two articles were added by hand searching of related review. After removing 93 duplicates, a total of 1739 articles were taken into next step. By screening on title and abstract, 1714 articles that did not meet the criteria were excluded. Although 22 fulltext articles were assessed for eligible, 9 were excluded due to various reasons (Fig. 1). Finally, 13 eligible studies were adopted for further data extraction [7, 12, 18-28].

\section{Characteristics of included studies}

The basic characteristics of all 13 eligible studies in this NMA are listed in Table 1. Overall, 1384 patients with congenital heart disease undergoing surgery were involved. In this NMA, 11 studies were two-arm studies and 2 were three-arm studies.
The average age of patients is 21.2 months and the average weight is $9.3 \mathrm{~kg}$. The majority of the eligible trials were based on randomized controlled trial, while only three were retrospective studies and one was unclear. Moreover, the relationship between each two interventions is plotted in Fig. 2.

\section{Network meta-analysis}

As presented by Fig. 3 and Table 2, in term of VT, the MD of drug treatments were lower than that of saline, which indicated that the all the drugs therapies showed better efficacy than saline. But the differences were not obvious statistically except dexmedetomidine. What is worth mentioned is that using fentanyl alone performs to be the worst but still superior to saline (MD $=4.84,95 \% \mathrm{CrIs}-2.47$ to 13.65$)$. The similar outcomes could also be found in terms of intensive care unit time that the dexmedetomidine showed a statistical advantage compared with saline $(\mathrm{MD}=1.26,95 \%$ CrIs 0.11-2.45). When it comes to LOS, mortality and JET (Fig. 4), the drug treatments, such as dexmedetomidine, fentanyl combined with dexmedetomidine and fentanyl alone, all achieved better efficacies than saline except fentanyl in JET (OR $=0.93,95 \%$ CrIs 0.016-30), and the differences of dexmedetomidine in JET (OR $=4.1,95 \%$ CrIs $1.3-17)$, fentanyl combined with dexmedetomidine $(\mathrm{OR}=3.3 \mathrm{e}+07,95 \%$ CrIs $1.8-5.7 e+21)$ and fentanyl alone $(\mathrm{OR}=2.7 \mathrm{e}+07,95 \%$ CrIs $1.7-4.3 e+21)$ in mortality were statistically significant.

\section{Consistency assessments}

In this study, net heat plots (Fig. 5) were used to assess the consistency level. And the outcomes indicated that no significant discrepancy was found among all the outcomes. 


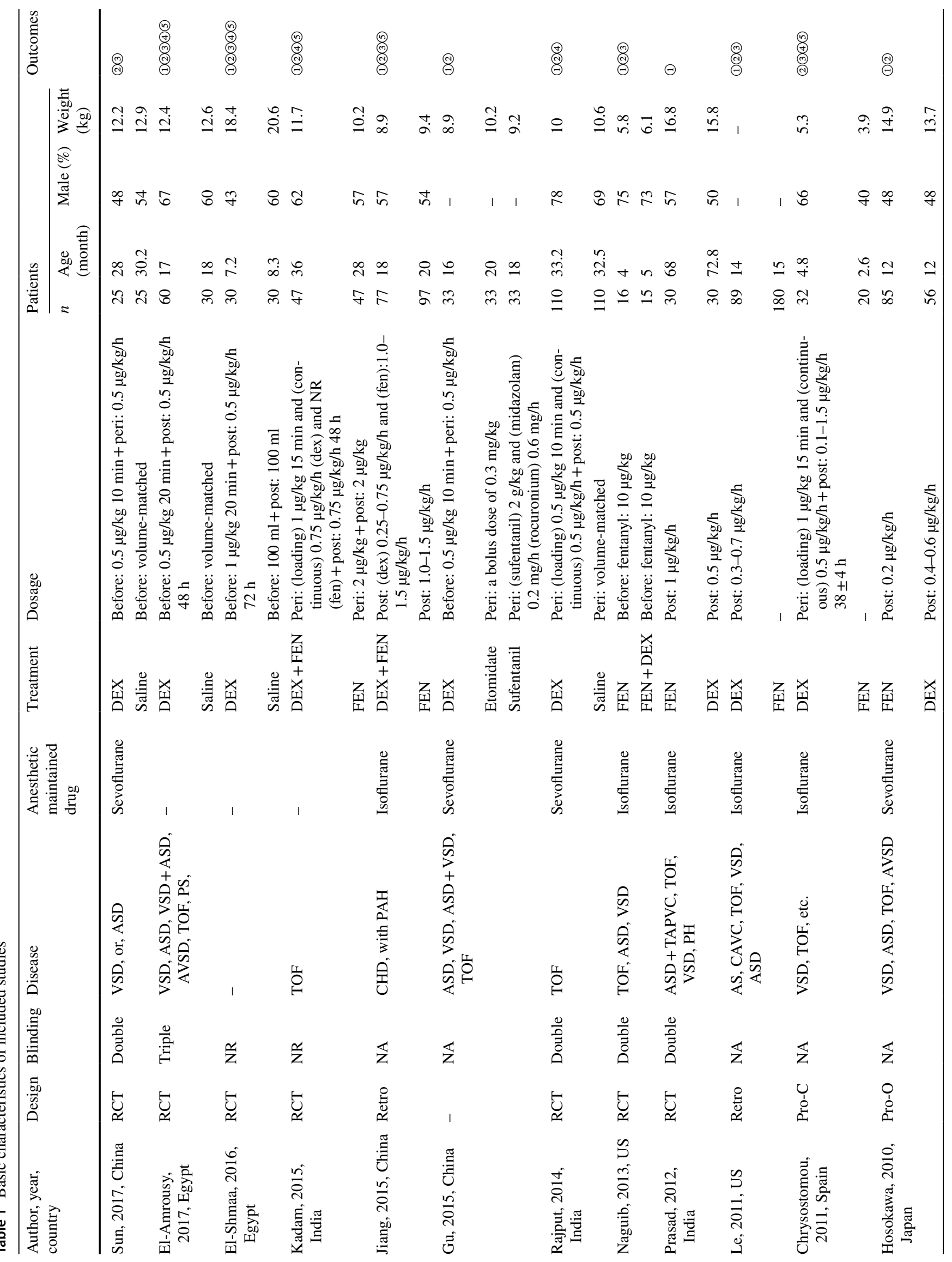




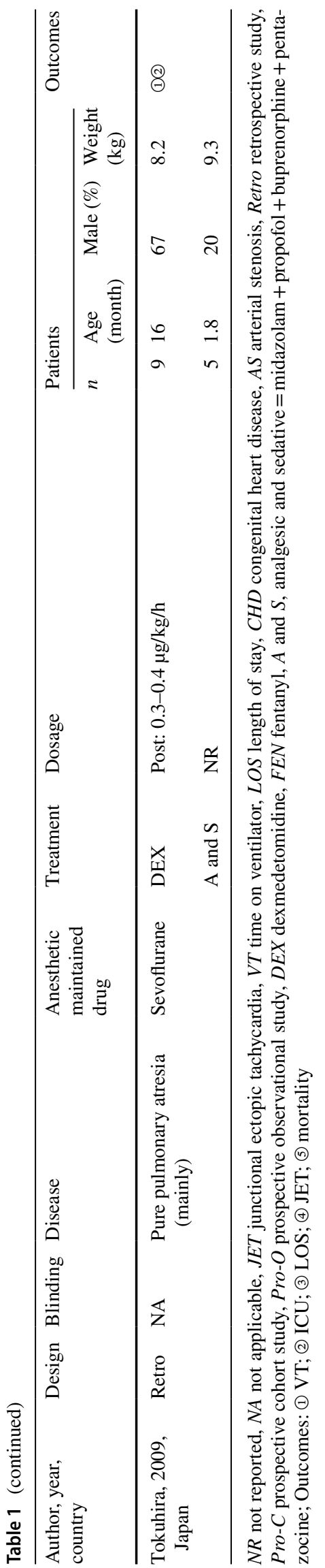

\section{Ranking with SUCRA value}

The ranking probabilities and SUCRA values of each treatment in terms of each endpoint are illustrated in Table 3. As for the efficacy outcomes, fentanyl combined with dexmedetomidine $(0.575)$ and etomidate $(0.589)$ performed better than other treatments in VT. And fentanyl combined with dexmedetomidine seemed to be the most effective one among others treatments under the endpoints of mortality (0.627). In the point of ICU time, analgesic and sedative showed a great potential to rank in the first place $(0.615)$. As for LOS, dexmedetomidine, fentanyl, and dexmedetomidine combined with fentanyl showed similar efficacy $(0.459,0.482$ and 0.497 , respectively). Surprisingly, the efficacy of saline seemed to be better than fentanyl in JET. And saline is the worst one under the other efficacy outcomes.

\section{Risk of bias}

The comparison adjusted funnel plots in Fig. S1. According to the criteria of bias, no obvious publication bias was found in this NMA.

\section{Discussion}

Based on our analysis, in terms of the respective efficacy for different drug therapies, all drugs have been demonstrated to be superior clinical effective compared to saline except fentanyl alone in JET. Fentanyl combined with dexmedetomidine, etomidate and sufentanil appeared to be the preferable drugs when the VT was taken at the first consideration. In terms of ICU time and JET, analgesic and sedative and dexmedetomidine achieved at the leading position, respectively. From the point of LOS, dexmedetomidine, fentanyl combined with dexmedetomidine or not performed no statistical differences with each other and all showed better efficacy than saline. As for mortality, fentanyl combined with dexmedetomidine had optimal efficacy.

Direct comparisons are the best way to evaluate the effectiveness among different drugs. However, due to scare of these head-to-head comparisons, one of the strength way is to enforce the method of NMA. Although various treatments have been assessed in previous MA, many of them just involve few of the treatments. Prasad et al., Le et al., Chrysostomou et al. and Tokuhira et al. all compared the efficacy between fentanyl and dexmedetomidine [18, 22, 24, 26]. However, the endpoints reported in these four studies varied. Prasad et al. only reported VT while Chrysostomou et al. studied the ICU time, LOS, JET and mortality but no VT $[18,26]$. Such problem would make it difficult for us to compare the therapeutic effect between fentanyl and 
Fig. 2 Evidence network. The width of the lines represents the cumulative number of trials for each comparison; the area of circles stands for the cumulative number of patients for each intervention $V T$ time on ventilator (h), ICU time in intensive care unit (d), LOS length of stay (d), JET junctional ectopic tachycardia
VT (h)

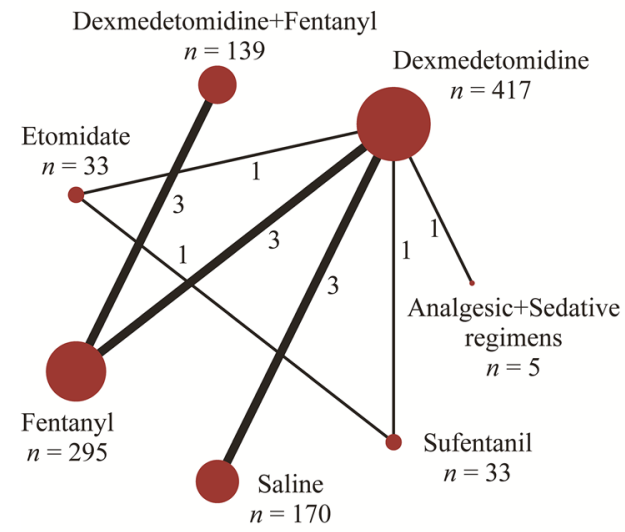

$\operatorname{LOS}(d)$



Mortality



ICU (d)

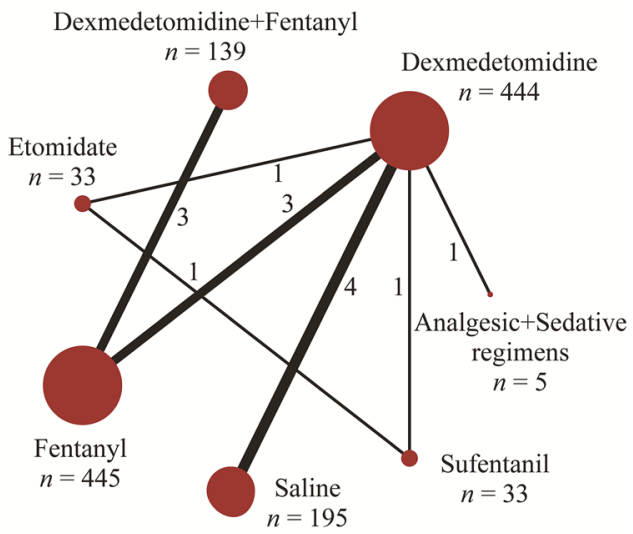

JET

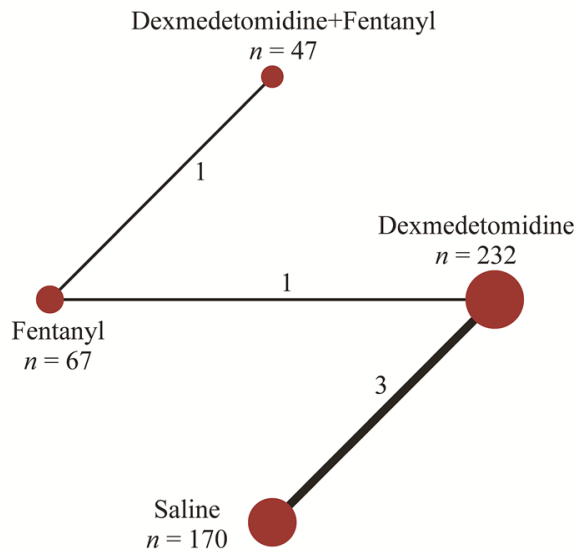

dexmedetomidine comprehensively. On the other hand, limited by the design and feasibility, one study tend to investigate only one or two drugs during one period. Sun et al., El-Amrousy et al. and Rajput et al. investigated the efficacy of dexmedetomidine in contrast to saline [19, 27, 28]. Kadam et al., Jiang et al. and Naguib et al. studied the outcomes after using fentanyl alone or fentanyl combined with dexmedetomidine $[7,23,25]$. In this NMA, we combined and analyzed the efficacy of all the frequently used drugs which were mentioned in previous studies for pediatric patients with congenital heart disease undergoing surgery. All common drugs used to manage postoperative patients with CHD from previous control studies were analyzed in current NMA and the efficacy of them was ranked under the five endpoints (VY, ICU time, LOS, JET and mortality). Through this NMA, it is helpful for us to determine the appropriate choice of drugs for postoperative pediatric patients with CHD from multiple points of view. Thus, this 
VT



ICU

Compared with Analgesic+Sedative

Dexmedetomidine

Dexmedetomidine + Fentanyl

Etomidate

Fentanyl

Saline

Sufentanil

Compared with Dexmedetomidine

Dexmedetomidine+Fentanyl

Etomidate

Fentanyl

Saline

Sufentanil

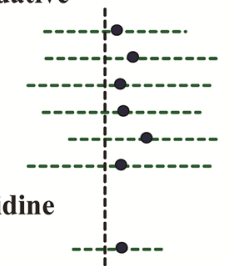

$\mathrm{MD}(95 \% \mathrm{CrI})$

$0.57(-2.44,3.55)$

$1.26(-2.38,4.86)$

$0.72(-3.14,4.60)$

$0.85(-2.49,4.16)$

$1.83(-1.38,5.06)$

$0.75(-3.13,4.62)$

$0.69(-1.35,2.71)$

$0.14(-2.33,2.62)$

$0.28(-1.21,1.74)$

$1.26(0.11,2.45)$

$0.19(-2.28,2.62)$

Compared with Dexmedetomidine+Fentanyl

Etomidate

Fentanyl

Saline

Sufentanil

Compared with Etomidate

Fentanyl

Saline

Sufentanil

Compared with Fentanyl

Saline

Sufentanil

Compared with Fentanyl

Sufentanil

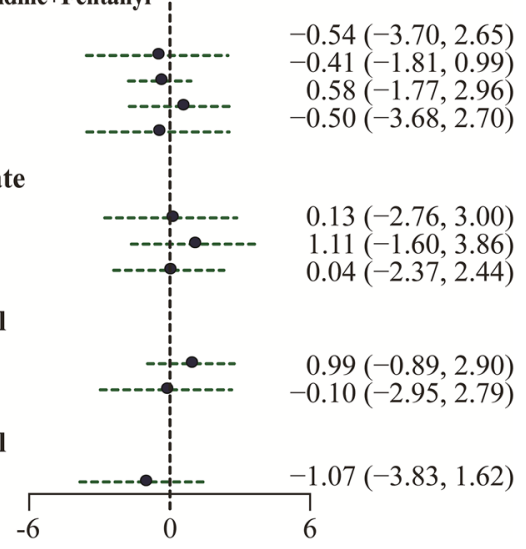

$-0.54(-3.70,2.65)$

$0.41(-1.81,0.99)$

$0.58(-1.77,2.96)$

$0.58(-1.77,2.96)$
$-0.50(-3.68,2.70)$

$0.13(-2.76,3.00)$

$1.11(-1.60,3.86)$

$0.99(-0.89,2.90)$

$-1.07(-3.83,1.62)$

Fig. 3 Forest plot. Mean differences with 95\% CrI showing the relative efficacy of medications under outcome of VT and ICU. VT time on ventilator, ICU Intensive Care Unit

study can be a guideline for the clinical workers when making decision.

However, when it comes to recommending one optimal drug, integrated factors also need to be considered. With respect to the different outcomes, fentanyl combined with dexmedetomidine was one of the recommended drugs due to its shorter time on ventilator and stay in hospital as well as lower mortality. Analgesic and sedative also showed an outstanding performance in terms of decreasing ICU time reducing the incidence of junctional ectopic tachycardia, and even seems better than fentanyl combined with dexmedetomidine. Besides, the investigation about etomidate and sufentanil was also in scarcity. Gu et al. compared dexmedetomidine, etomidate and sufentanil in a study with 3 contrasts included 33 in each group but the design of his study was not reported [21]. Given the decent outcomes of etomidate and sufentanil in this network meta-analysis, more research about these two drugs could be taken into consideration to provide more evidence for the use of etomidate and sufentanil.

However, despite the improvements achieved in this NMA, there are still some limitations in our study. Our study is limited to using VT, ICU time, LOS, JET and mortality as the endpoints. While, not all the studies included in this NMA have reported all these five results. The lack of specific outcomes values in some original studies makes it difficult to compare all drugs under those endpoints. For example, etomidate and sufentanil and analgesic and sedative were missing under the endpoints of LOS, JET and mortality. Another potential limitation is the doses of the treatments which do not considered in this study. Given small sample size and lack of sufficient raw data, the dosing and timing have not included to analysis. Therefore, the optimal dosing, timing, and duration of different drugs was not available from this NMA. In addition, as for the reliability of NMA, between-study heterogeneity, such as average age, MRI, male ratio, history treatments were also influential. Moreover, since different outcomes were chosen by eligible trials and multiple treatments involved in this NMA, it is hard to compare all treatments under one endpoints and this would increase the consistency. However, according to our analysis, inconsistency was not found in this NMA. All in all, to resolve all the restrictions mentioned above, betterdesigned RCTs need be conducted.

Overall, based on the comprehensive consideration of efficacy, fentanyl combined with dexmedetomidine was considered the best-recommended clinical interventions among integrated endpoints. Even though this study provides some 


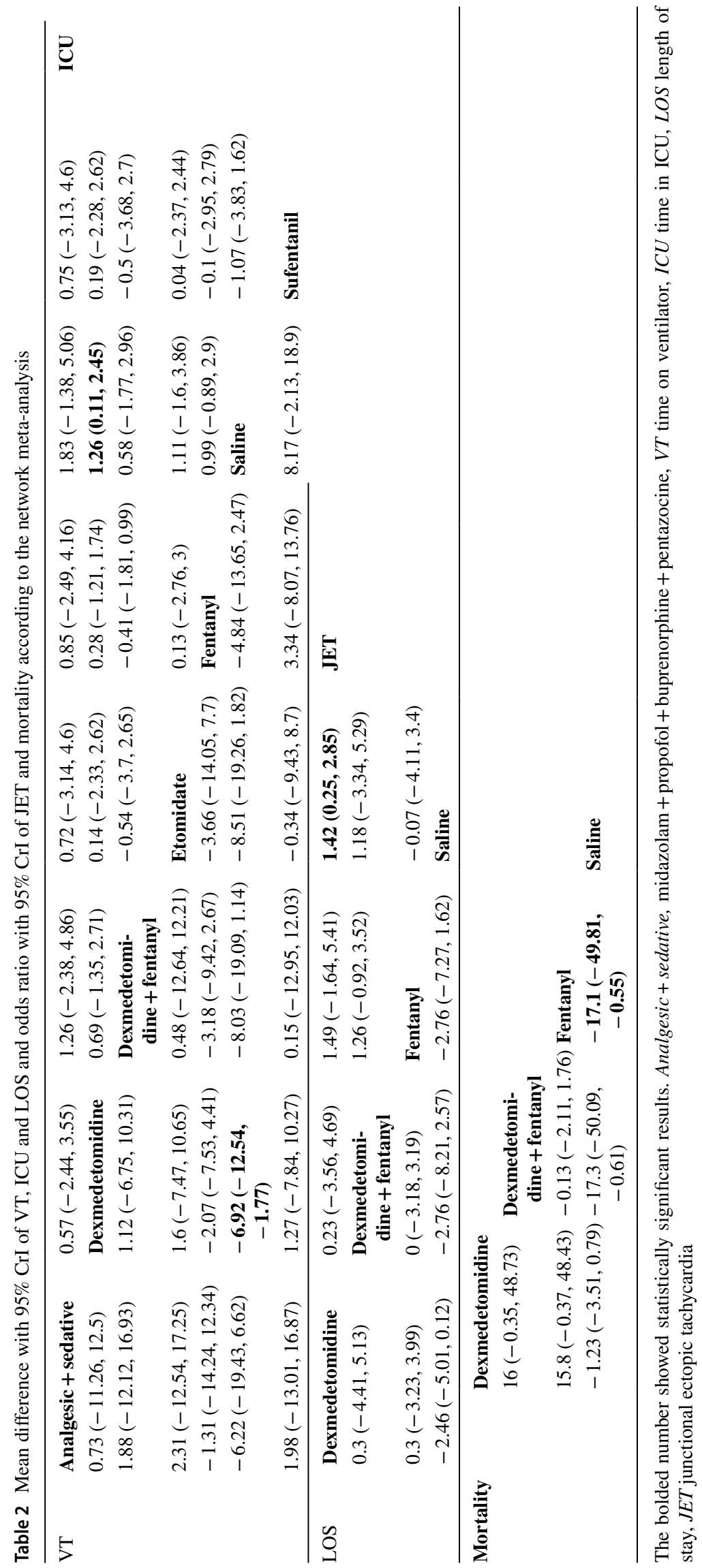




\section{LOS}

Compared with Dexmedetomidine

Dexmedetomidine+Fentanyl -------------

Fentanyl

Saline

Compared with Dexmedetomidine

+Fentanyl

Fentanyl

Saline

Compared with Fentanyl

Saline

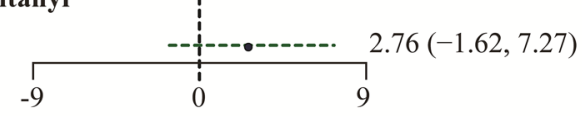

MD $(95 \% \mathrm{CrI})$

$-0.30(-5.13,4.41)$

$-0.30(-3.99,3.23)$

$2.46(-0.12,5.01)$

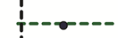

Compared with Dexmedetomidine

Dexmedetomidine

+ Fentanyl

Fentanyl

Saline

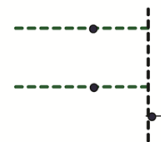

OR $(95 \% \mathrm{CrI})$

$1.1 \mathrm{e}^{-07}(6.8 \mathrm{e}-22,1.4)$

$1.4 \mathrm{e}^{-0} 07(9.2 \mathrm{e}-22,1.4)$

$3.4(0.46,34)$

Compared with Dexmedetomiding

+Fentanyl

Fentanyl

$1.1(0.17,8.3)$

Saline

$-3.3 \mathrm{e}+07(1.8,5.7 \mathrm{e}+21)$

Compared with Fentanyl

Saline

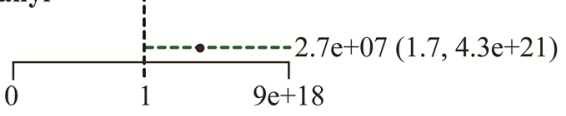

Fig. 4 Forest plot. Mean differences with 95\% CrI showing the relative efficacy of medications under outcome of LOS and odd ratios with 95\% CrI of mortality and JET. JET junctional ectopic tachycardia, LOS length of stay


Fig. 5 Heat plot. Net heat plots for different study designs revealing inconsistency between direct and indirect evidence. $A$ sufentanil, $B$ saline, $C$ fentanyl, $D$ etomidate, $E$ dexmedetomidine + fentanyl, $F$ dexmedetomidine, $G$ analgesic + sedative 
Table 3 SUCRA values of 7 treatments

\begin{tabular}{llllll}
\hline Treatments & VT & ICU & LOS & JET & Mortality \\
\hline Analgesic + sedative & 0.431 & $\mathbf{0 . 6 1 5}$ & - & - & - \\
Dexmedetomidine & 0.485 & $\mathbf{0 . 5 5 3}$ & 0.459 & $\mathbf{0 . 5 9 3}$ & 0.238 \\
Dexmedetomidine+fen- & $\mathbf{0 . 5 7 5}$ & 0.307 & $\mathbf{0 . 4 8 2}$ & $\mathbf{0 . 5 1 7}$ & $\mathbf{0 . 6 2 7}$ \\
$\quad$ tanyl & & & & & \\
Etomidate & $\mathbf{0 . 5 8 9}$ & 0.473 & - & - & - \\
Fentanyl & 0.304 & 0.455 & $\mathbf{0 . 4 9 7}$ & 0.188 & $\mathbf{0 . 5 9 8}$ \\
Saline & 0.053 & 0.135 & 0.062 & 0.202 & 0.036 \\
Sufentanil & 0.563 & 0.463 & - & - & - \\
\hline
\end{tabular}

Top two SUCRA under each endpoint were bolded. Analgesic + sedative midazolam + propofol + buprenorphine + pentazocine, $V T$ time on ventilator, ICU time in ICU, LOS length of stay, JET junctional ectopic tachycardia

comparison for the efficacy and effectiveness of various drugs, clinicians still need to consider the side effects and safety of the therapy, clinical preference, patients' feature, and disease history to choose the suitable treatments.

Author contributions RL conceived and coordinated the study, designed, performed and analyzed the experiments, and wrote the paper. BL and GZ carried out the data collection, data analysis, and revised the paper. All the authors reviewed the results and approved the final version of the manuscript.

Funding None.

\section{Compliance with ethical standards}

Ethical approval This article does not contain any studies with human participants or animals performed by any of the authors.

Conflict of interest No financial or nonfinancial benefits have been received or will be received from any party related directly or indirectly to the subject of this article.

\section{References}

1. Benjamin EJ, Virani SS, Callaway CW, Chamberlain AM, Chang AR, Cheng S, et al. Heart disease and stroke statistics-2018 update: a report from the American Heart Association. Circulation. 2018;137:e67-492.

2. Lloyd-Jones D, Adams RJ, Brown TM, Carnethon M, Dai S, De Simone G, et al. Heart disease and stroke statistics-2010 update: a report from the American Heart Association. Circulation. 2010;121:e46-215.

3. Welke KF, Shen I, Ungerleider RM. Current assessment of mortality rates in congenital cardiac surgery. Ann Thorac Surg. 2006;82:164-70 (discussion 170-61).

4. Galante D, Meola S, Pellico G, Tancredi ML, Milillo R. Anesthetic management of children with pulmonary arterial hypertension. Paediatr Anaesth. 2008;18:562-3 (author reply 563-4).
5. Fischer LG, Van Aken H, Burkle H. Management of pulmonary hypertension: physiological and pharmacological considerations for anesthesiologists. Anesth Analg. 2003;96:1603-16.

6. Koruk S, Mizrak A, Kaya Ugur B, Ilhan O, Baspinar O, Oner U. Propofol/dexmedetomidine and propofol/ketamine combinations for anesthesia in pediatric patients undergoing transcatheter atrial septal defect closure: a prospective randomized study. Clin Ther. 2010;32:701-9.

7. Jiang L, Ding S, Yan H, Li Y, Zhang L, Chen X, et al. A retrospective comparison of dexmedetomidine versus midazolam for pediatric patients with congenital heart disease requiring postoperative sedation. Pediatr Cardiol. 2015;36:993-9.

8. Freire AX, Afessa B, Cawley P, Phelps S, Bridges L. Characteristics associated with analgesia ordering in the intensive care unit and relationships with outcome. Crit Care Med. 2002;30:2468-72.

9. Riker RR, Fraser GL. Adverse events associated with sedatives, analgesics, and other drugs that provide patient comfort in the intensive care unit. Pharmacotherapy. 2005;25:8s-18s.

10. Belleville JP, Ward DS, Bloor BC, Maze M. Effects of intravenous dexmedetomidine in humans. I. Sedation, ventilation, and metabolic rate. Anesthesiology. 1992;77:1125-33.

11. Triltsch AE, Welte M, von Homeyer P, Grosse J, Genahr A, Moshirzadeh M, et al. Bispectral index-guided sedation with dexmedetomidine in intensive care: a prospective, randomized, double blind, placebo-controlled phase II study. Crit Care Med. 2002;30:1007-14.

12. Tokuhira N, Atagi K, Shimaoka H, Ujiro A, Otsuka Y, Ramsay M. Dexmedetomidine sedation for pediatric post-Fontan procedure patients. Pediatr Crit Care Med. 2009;10:207-12.

13. Zampi JD, Hirsch JC, Gurney JG, Donohue JE, Yu S, LaPage MJ, et al. Junctional ectopic tachycardia after infant heart surgery: incidence and outcomes. Pediatr Cardiol. 2012;33:1362-9.

14. Andreasen JB, Johnsen SP, Ravn HB. Junctional ectopic tachycardia after surgery for congenital heart disease in children. Intensive Care Med. 2008;34:895-902.

15. Ghimire LV, Chou FS. Efficacy of prophylactic dexmedetomidine in preventing postoperative junctional ectopic tachycardia in pediatric cardiac surgery patients: a systematic review and metaanalysis. Paediatr Anaesth. 2018;28:597-606.

16. Liu Y, Bian W, Liu P, Zang X, Gu X, Chen W. Dexmedetomidine improves the outcomes in paediatric cardiac surgery: a meta-analysis of randomized controlled trials. Interact Cardiovasc Thorac Surg. 2018;26:852-8.

17. Pan W, Wang Y, Lin L, Zhou G, Hua X, Mo L. Outcomes of dexmedetomidine treatment in pediatric patients undergoing congenital heart disease surgery: a meta-analysis. Paediatr Anaesth. 2016;26:239-48.

18. Chrysostomou C, Sanchez-de-Toledo J, Wearden P, Jooste EH, Lichtenstein SE, Callahan PM, et al. Perioperative use of dexmedetomidine is associated with decreased incidence of ventricular and supraventricular tachyarrhythmias after congenital cardiac operations. Ann Thorac Surg. 2011;92:964-72 (discussion 972).

19. El Amrousy DM, Elshmaa NS, El-Kashlan M, Hassan S, Elsanosy M, Hablas N, et al. Efficacy of prophylactic dexmedetomidine in preventing postoperative junctional ectopic tachycardia after pediatric cardiac surgery. J Am Heart Assoc. 2017;6:e004780.

20. El-Shmaa NS, El Amrousy D, El Feky W. The efficacy of preemptive dexmedetomidine versus amiodarone in preventing postoperative junctional ectopic tachycardia in pediatric cardiac surgery. Ann Card Anaesth. 2016;19:614-20.

21. Gu H, Zhang M, Cai M, Liu J. Comparison of adrenal suppression between etomidate and dexmedetomidine in children with congenital heart disease. Med Sci Monit. 2015;21:1569-76.

22. Hosokawa K, Shime N, Kato Y, Taniguchi A, Maeda Y, Miyazaki $\mathrm{T}$, et al. Dexmedetomidine sedation in children after cardiac surgery. Pediatr Crit Care Med. 2010;11:39-43. 
23. Kadam SV, Tailor KB, Kulkarni S, Mohanty SR, Joshi PV, Rao SG. Effect of dexmeditomidine on postoperative junctional ectopic tachycardia after complete surgical repair of tetralogy of Fallot: a prospective randomized controlled study. Ann Card Anaesth. 2015;18:323-8.

24. Le KN, Moffett BS, Ocampo EC, Zaki J, Mossad EB. Impact of dexmedetomidine on early extubation in pediatric cardiac surgical patients. Intensive Care Med. 2011;37:686-90.

25. Naguib AN, Tobias JD, Hall MW, Cismowski MJ, Miao Y, Barry $\mathrm{N}$, et al. The role of different anesthetic techniques in altering the stress response during cardiac surgery in children: a prospective, double-blinded, and randomized study. Pediatr Crit Care Med. 2013;14:481-90.
26. Prasad SR, Simha PP, Jagadeesh AM. Comparative study between dexmedetomidine and fentanyl for sedation during mechanical ventilation in post-operative paediatric cardiac surgical patients. Indian J Anaesth. 2012;56:547-52.

27. Rajput RS, Das S, Makhija N, Airan B. Efficacy of dexmedetomidine for the control of junctional ectopic tachycardia after repair of tetralogy of Fallot. Ann Pediatr Cardiol. 2014;7:167-72.

28. Sun Y, Liu J, Yuan X, Li Y. Effects of dexmedetomidine on emergence delirium in pediatric cardiac surgery. Minerva Pediatr. 2017;69:165-73.

Publisher's Note Springer Nature remains neutral with regard to jurisdictional claims in published maps and institutional affiliations. 